\title{
Medical Ethnobiology and Indigenous Knowledge System of the Lapcha of Fikkal VDC of Ilam, Nepal
}

\author{
Pradip Tamang ${ }^{1}$ and Nanda B. Singh ${ }^{2}$ \\ Central Department of Zoology, Tribhuvan University, Kirtipur, Kathmandu \\ ${ }^{1}$ E-mail: lama.pradip7@gmail.com) and 2E-mail:nanda_nepal@yahoo.com
}

\begin{abstract}
The main aim of this paper is to investigate the medico-ethnobiological information along with indigenous knowledge system of Lapchas, one of the endangered indigenous nationalities of Nepal from Fikkal VDC of Ilam district. They were found to have rich and profound indigenous knowledge on the use of plants and animals for various purposes. Analysis of data revealed that local Lapcha people had a rich tradition on the usage of animal species and plant species of medicinal values for medicinal use for their primary health care services. Different medicinal plant and animal species were used for the treatment of different disease/ailments. The respiratory tract infections, gastrointestinal disorders, skeleto-muscular problems and dermatological infections were the most frequent ailments/diseases treated. The findings revealed by the present study have also been supported by the works previously done on other part of the country.
\end{abstract}

Keywords: Indigenous knowledge, traditional medicine, medicinal animals and plants, Lapcha, ethnic community.

\section{INTRODUCTION}

Nepal is a wonderland with limitless beauty embosomed in the world's mightiest mountain chains the Himalayas, stretching from tropical plains to stupendous snow heights. Owing to this diverse geography and wide range of altitudinal and climatic zones, she has magnificent, rich and varied biological resources. Along with the diverse floral and faunal resources, she also endows an array of ethnic groups rich in tradition, culture and indigenous knowledge system. The indigenous knowledge present in people from time immemorial has helped in the sustainable utilization of biological resources for various purposes like food, medicine, clothing, dyes, construction etc. The indigenous knowledge for the utilization of plants and animals' parts as medicine has been the only way to save life of the people in the rural area of Nepal where modern health facilities is still out of reach. Still $87 \%$ of people living in rural area of Nepal depend directly or indirectly on the formal and informal system of traditional medicines for healthcare (Bhattarai, 1992, CBS, 2011). So, use of traditional medicine in remote areas of Nepal is a necessity than a luxury.

In Nepal, the use of plants and animals' parts as medicine is widespread. In rural areas of Nepal, medicinal knowledge and practices are passed down entirely through the oral, traditional and personal experiences, keeping alive the rich traditional health care system like Ayurveda, Amchi, Homeopathy associating with Unani, and Naturopathy. "Medicinal Plants of Nepal", a book published by the Department of Medicinal plants in two volumes (HMGN, 1970, 1984) accounted 571 species from Nepal. Malla and Shakya (1984-1985) compiled a list of 630 species of medicinal plants from Nepal, out of these 510 species are indigenous i.e. they occur in wild and 120 species are either exotic or indigenous that are naturalized or cultivated since long. However, around 1000 species of medicinal and aromatic plants have been estimated to occur in Nepal (Chaudhary, 1998). These plants occur throughout the country from tropical forest to alpine meadows. The work of Malla and Shakya (19841985) revealed the presence of maximum number of medicinal plant species, 540 (i.e. 85\%) in central Nepal. The numbers from Western and Eastern regions are 424 $(67.3 \%)$ and $512(81.27 \%)$ respectively. Around 1403 species have been reported which represents about $20 \%$ of the total estimated flowering plants of Nepal (Tiwari, 1994). The indigenous people living close to nature have a sound knowledge on the usage of these plants and animals for different medicinal purposes.

Lapcha, one of the ten endangered indigenous nationalities of Nepal comprising around 2 per 10,000 populations of Nepal (CBS, 2003, 2011) that accounts for one of the least populated indigenous group by "National Foundation for Development of Indigenous Nationalities Act, 2058 (2002 AD)". They are found mostly concentrated in Ilam district of eastern Nepal. Most Lapcha in Nepal are peasants: small subsistence formers trying to get what they can produce on their small plots of land situated near the forest or forest patches. Because of their close association with nature, they have abundant knowledge and skill in utilizing natural forest products found in their vicinity for traditional medicinal practices. In this context, the main objectives of this paper are: (i) to 
provide medico-ethnobiological information, and (ii) to explore the pristine indigenous knowledge system found in the Lapcha.

\section{MATERIALS AND METHODS}

\section{Study area}

Ilam district also known as the 'queen of hills' lies in the Mechi Zone at eastern boundary of Nepal touching the Darjeeling district of India in the east, Morang and Dhankuta districts in the west, Panchthar district in the North and Jhapa district in the south. It lies within $26^{\circ} 40^{\prime}$ to $27^{\circ} 8^{\prime}$ latitude and $87^{\circ} 40^{\prime}$ to $88^{\circ} 10^{\prime}$ longitude having the area of $1,703 \mathrm{sq} . \mathrm{km}$. It lies at the altitudinal range of $600-3700 \mathrm{~m}$ from the sea level. The district headquarter of Ilam is Ilam bazaar.

Fikkal is one the 48 VDCs of Ilam district which is located at an altitude of $1500 \mathrm{~m}$ above sea level, with an area of 27 sq.km. The VDC boundary of Fiklal VDC is Shri Antu on the east, Kanyam on south east, Pashupatinagar on north east, Gorkhe on north, Pachakanya on west and Naya bazar on north west. Along with the Lapcha community, different other ethnic/caste groups also inhabit the area like Limbu, Rai, Tamang, Brahmin, Chhetri, Newar etc. However, a fairly large population of Lapcha resides in this VDC, so the study is focused on this village development committee.
To accomplish above mentioned objectives, primary and secondary sources of data were used. Primary data were collected from the field visit by direct observation and questionnaire methods. Key-informants for traditional medicinal practices like the local healers like Dhami, Jhankri, Boonthing/Mun, and Yaba/Yama were asked a number of structured and semi-structured questions about traditional healing practices and the plants and animals used as medicine for different diseases. Unstructured interview with small group comprising youths, women, and local elderly people was also done to generate information. Samples of different animals and plants both known and unknown were collected from the field visit which was done twice in the month of October 2007 and June 2008.

The collected samples were identified with standard literatures and with the help of experts (Botanist and Zoologist) from the Central Department of Zoology and Botany (T.U.) and the National Herbarium, Godawari, Kathmandu. The plants and animal species were taxonomically classified into division, order, family, genera and species. The secondary data were colleted from the relevant textbooks, research paper, journals and publications which helped in comparison and justification of primary data.

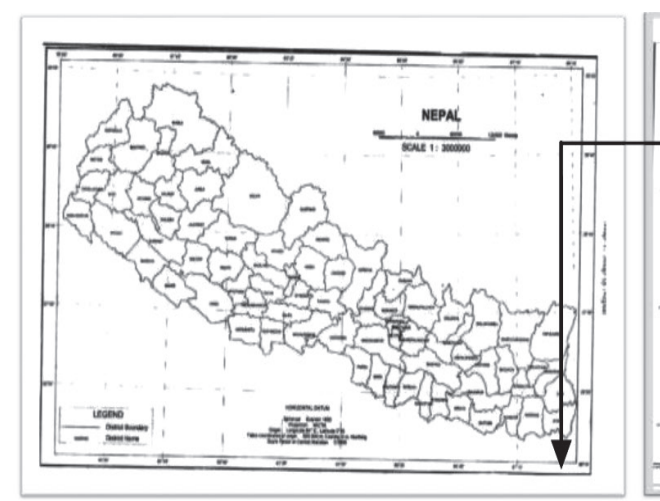

Fig. 1. Map of Nepal

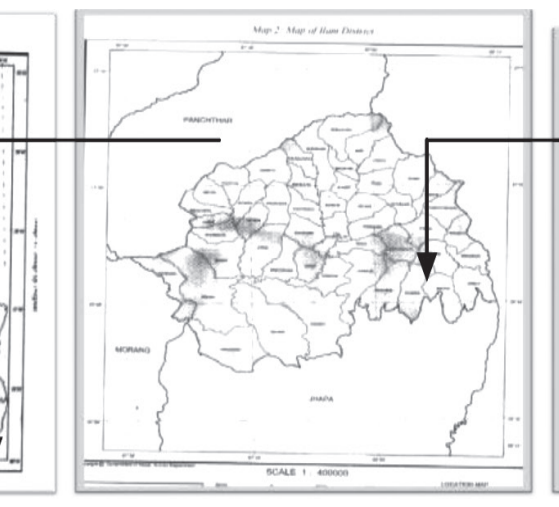

Fig. 2. Map of Ilam district

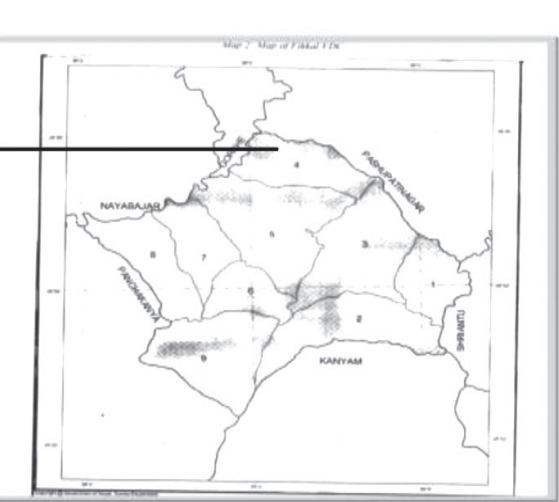

Fig. 3. Map of Fikkal VDC

\section{RESULTS}

The result has been presented into two categories of medico-ethnobiological and indigenous knowledge system as below.

\section{Ethnomedical biology}

Lapcha community of Fikkal VDC have been using a number of animal species both wild and domesticated in their traditional healing system as medicines to cure several diseases. Findings of this research disclosed that altogether 19 animal species both wild and domesticated, belonging to 10 order 13 families have been used for the treatment of 21 different diseases/ailments. It was found that the Lapcha people have profound knowledge about different plant species having medicinal value used in their traditional healing practices and they make use of some 61 species of medicinal plants belonging to 39 families and 58 genera for curing 36 different ailments by using their own indigenous knowledge. The list of animals and plants species used in the traditional medicine by the local Lapcha people of the study area is shown in Table 1. 

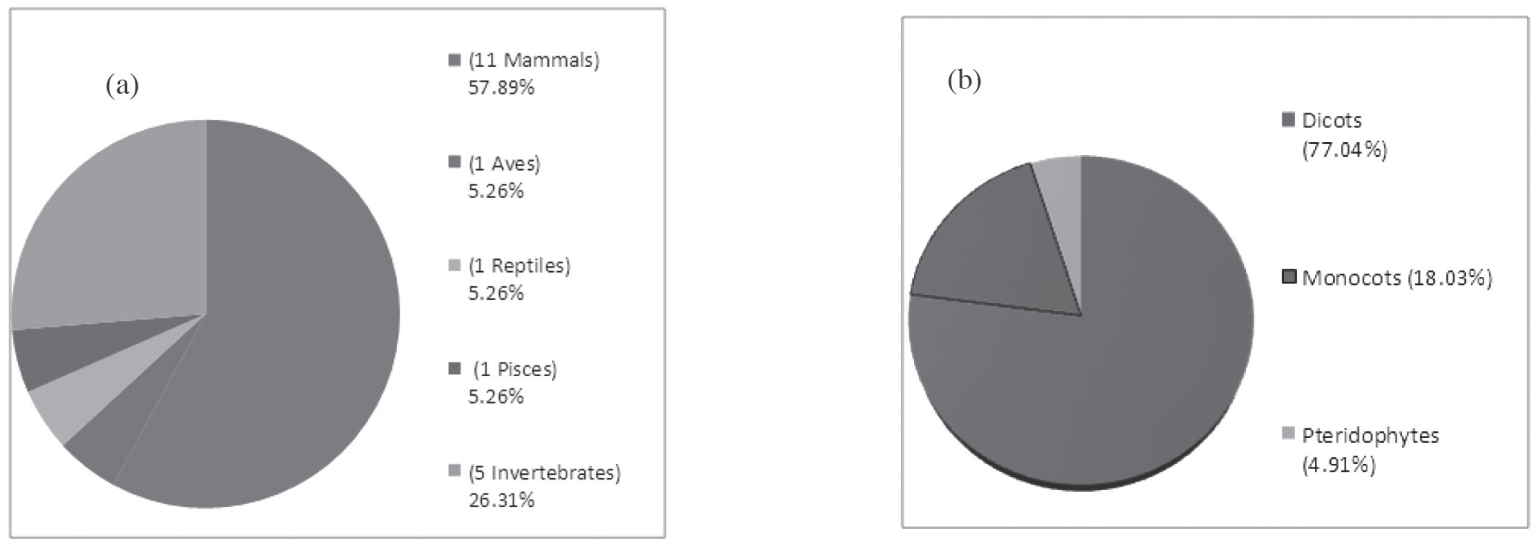

Fig. 4. Medico-ethnobiological use of Lapcha (a) animal category and (b) plant category

Table 1. Categorization of Animal Species Used in Folk Medicines by the local Lapcha of Fikkal VDC, Ilam.

\begin{tabular}{|c|c|c|c|c|c|c|c|c|}
\hline S.N. & Order & Family & $\begin{array}{l}\text { Scientific } \\
\text { Name }\end{array}$ & Type & $\begin{array}{l}\text { Local } \\
\text { Name }\end{array}$ & $\begin{array}{l}\text { English } \\
\text { Name }\end{array}$ & $\begin{array}{c}\text { Organ } \\
\text { Used }\end{array}$ & Medicinal Uses \\
\hline 1. & Aritiodactyla & Bovidae & $\begin{array}{l}\text { Bubalus } \\
\text { bubalus }\end{array}$ & Mammal & Bhaisi & Buffalo & $\begin{array}{l}\text { Tooth, } \\
\text { Bile }\end{array}$ & Boils, Asthma \\
\hline 2. & Artiodactyla & Bovidae & Bos indicus & Mammal & Gai & Cow & $\begin{array}{l}\text { Tooth, } \\
\text { milk }\end{array}$ & $\begin{array}{l}\text { Boils, Back and Body } \\
\text { pain }\end{array}$ \\
\hline 3. & Artiodactyla & Bovidae & Capra hiscus & Mammal & Bakhra & Goat & Bile & Asthma \\
\hline 4. & Artiodactyla & Cervidae & Axis axis & Mammal & Harin & Deer & $\begin{array}{l}\text { Hoof and } \\
\text { Foetus }\end{array}$ & Mushroom Poison. \\
\hline 5. & Artiodactyla & Suidae & Sus sp. & Mammal & Sungur & Pig & Bile & Asthma \\
\hline 6. & Anura & Ranidae & Rana tigrina & Amphibia & Manpawa & $\begin{array}{l}\text { Indian } \\
\text { Bull Frog }\end{array}$ & $\begin{array}{l}\text { Meat and } \\
\text { Egg }\end{array}$ & Dysentery and Pile \\
\hline 7. & Carnivora & Canidae & Canis familiar & Mammal & Kukur & Dog & Brain & Rabies. \\
\hline 8. & Carnivora & Canidae & Canis aureus & Mammal & Syal & Jackal & $\begin{array}{l}\text { Meat, } \\
\text { Bone and } \\
\text { Hide }\end{array}$ & Rheumatism and T.B. \\
\hline 9. & Carnivora & Felidae & Panthera tigris & Mammal & Bagh & Tiger & Bone & Baaghe. \\
\hline 10. & Decapoda & Palaemonidae & $\begin{array}{l}\text { Palaemon } \\
\text { malcolmsoni }\end{array}$ & Crustacean & $\begin{array}{l}\text { Jhinge } \\
\text { Macha }\end{array}$ & prawn & Mandible & Throne prick \\
\hline 11. & Galliformes & Phasinidae & $\begin{array}{l}\text { Gallus gallus } \\
\text { domesticus }\end{array}$ & Aves & Kukhura & Rooster & Egg, Fat & $\begin{array}{l}\text { Burnt wound, } \\
\text { Eczema, Body Pain. }\end{array}$ \\
\hline 12. & Hymenoptera & Apidae & Apis cerana & Insect & Gharmauri & $\begin{array}{l}\text { Honey } \\
\text { Bee }\end{array}$ & Honey & $\begin{array}{l}\text { Cough, Back and } \\
\text { Body pain. }\end{array}$ \\
\hline 13. & Hymenoptera & Apidae & Apis dorsata & Insect & Putka & $\begin{array}{l}\text { Honey } \\
\text { Bee }\end{array}$ & Honey & $\begin{array}{l}\text { Snake bite, Vomit, } \\
\text { Fatigue and loss of } \\
\text { appetite }\end{array}$ \\
\hline 14. & Perrisodactyla & Equidae & Equus coballus & Mammal & Ghoda & Horse & Milk & Thangne Biram \\
\hline 15. & Robocidca & Elephantidae & $\begin{array}{l}\text { Elephus } \\
\text { maximus }\end{array}$ & Mammal & Hatti & Elephant & Bone & Internal Eczema \\
\hline 16. & Rodentia & Hystricidae & $\begin{array}{l}\text { Hystric } \\
\text { brachyura }\end{array}$ & Mammal & Dumsi & Porcupine & Stomach & Asthma \\
\hline 17. & Stylomatophora & Helicidae & Anadenus sp. & Mollusca & Chiplekira & Slug & $\begin{array}{l}\text { Saliva, } \\
\text { whole } \\
\text { body }\end{array}$ & $\begin{array}{l}\text { Cut wound } \\
\text { Ringworm, Bone } \\
\text { fracture }\end{array}$ \\
\hline 18. & & & & Reptile & Sarpa & Snake & Bile & Cut wound. \\
\hline 19. & & & & Pisces & Macha & Fish & Fat & Burnt wound. \\
\hline
\end{tabular}


Medical Ethnobiology and Indigenous Knowledge System of the Lapcha of Fikkal VDC of Ilam, Nepal

Table 2. Categorization of Plant Species Used in Folk Medicine by the local Lapcha of Fikkal VDC, Ilam.

\begin{tabular}{|c|c|c|c|c|c|c|c|c|}
\hline S.N. & Division & Family & Scientific Name & \begin{tabular}{|l|} 
Local \\
Name \\
\end{tabular} & \begin{tabular}{|l|} 
Life \\
form
\end{tabular} & \begin{tabular}{|l|} 
Parts \\
Used \\
\end{tabular} & \begin{tabular}{|l|} 
Form of \\
medication
\end{tabular} & \begin{tabular}{|l|} 
Medicinal \\
Uses \\
\end{tabular} \\
\hline 1. & Dicotyledon & Apiaceae & $\begin{array}{l}\text { Heracleum } \\
\text { nepalense }\end{array}$ & Chimfin & $\begin{array}{l}\text { Small } \\
\text { tree }\end{array}$ & $\begin{array}{l}\text { Flower, } \\
\text { fruit }\end{array}$ & $\begin{array}{l}\text { Powder, } \\
\text { paste, Raw. }\end{array}$ & $\begin{array}{l}\text { Headache, } \\
\text { Stomach }\end{array}$ \\
\hline 2. & Dicotyledon & Asteraceae & $\begin{array}{l}\text { Ageratum } \\
\text { conyzoides }\end{array}$ & Ilame Jhar & Herb & Leaf & Juice & Insect bite \\
\hline 3. & Dicotyledon & Bignoniaceae & Orocylum indicum & Tatalo & Tree & Bark & Juice & Jaundice \\
\hline 4. & Dicotyledon & Brassicaceae & $\begin{array}{l}\text { Raphnus sativus } \\
\text { Linn }\end{array}$ & Banmula & Herb & Root & Paste, Raw & Burnt wound, \\
\hline 5. & Dicotyledon & Caryophyllaeceae & Drymaria Cordata & Abhijalo & Herb & Leaf & Juice, stem & $\begin{array}{l}\text { Common cold, } \\
\text { cut wound. }\end{array}$ \\
\hline 6. & Dicotyledon & Chenopodiaceae & $\begin{array}{l}\text { Chenopodium } \\
\text { album }\end{array}$ & Bethu & Herb & $\begin{array}{l}\text { whole } \\
\text { plant }\end{array}$ & Cooked & Constipation \\
\hline 7. & Dicotyledon & Compositae & Artemesia indica & Titepati & Herb & Leaf & $\begin{array}{l}\text { Power, } \\
\text { Juice, Paste }\end{array}$ & \begin{tabular}{|l} 
Nose bleeding, \\
Cut wound, \\
scabbies \\
\end{tabular} \\
\hline 8. & Dicotyledon & Compositae & Tagetes erecta L. & Sayapatri & Herb & Leaf & Decoction & Fever \\
\hline 9. & Dicotyledon & Cruciferae & $\begin{array}{l}\text { Brassica } \\
\text { Campestris }\end{array}$ & Tori & Herb & Seed & Oil (Raw) & $\begin{array}{l}\text { Back and } \\
\text { Body Pain } \\
\end{array}$ \\
\hline 10. & Dicotyledon & Cruciferae & Lepidium sativum & Chamsur & Herb & $\begin{array}{l}\text { Whole } \\
\text { plant, } \\
\text { seed }\end{array}$ & $\begin{array}{l}\text { Cooked, } \\
\text { Powder }\end{array}$ & $\begin{array}{l}\text { Body and back } \\
\text { pain, Bone } \\
\text { fracture. }\end{array}$ \\
\hline 11. & Dicotyledon & Cucurbitaceae & Cucumis stivus & Kankro & Climber & Fruit & Powder & Common cold. \\
\hline 12. & Dicotyledon & Diascoreaceae & Dioscorea sps. & Vyakur & Climber & Rhizome & Boiled & Constipation. \\
\hline 13. & Dicotyledon & tricaceae & $\begin{array}{l}\text { Rhododendron } \\
\text { arboretum }\end{array}$ & Laligurans & Tree & Flower & Raw & $\begin{array}{l}\text { Fish bone } \\
\text { prick }\end{array}$ \\
\hline 14. & Dicotyledon & Euphrobiacee & Emblica officinalis & Amala & Tree & Bark & Powder & Dysentery \\
\hline 15. & Dicotyledon & Fabiaceae & $\begin{array}{l}\text { Piptanthus } \\
\text { nepalensis }\end{array}$ & $\begin{array}{l}\text { Bakhre } \\
\text { lahara }\end{array}$ & Climber & Root & Powder & Gastric \\
\hline 16. & Dicotyleadon & Gentianaceae & Swertia Chiraita & Chiraito & Herb & \begin{tabular}{|l}
$\begin{array}{l}\text { Leaf, } \\
\text { stem }\end{array}$ \\
\end{tabular} & Decoction & Fever \\
\hline 17. & Dicotyledon & Labiatae & Mentha aquatica L. & Pudina & Herb & Leaf & Juice & Diarrhoea. \\
\hline 18. & Dicotyledon & Labiatae & $\begin{array}{l}\text { Ocimum basilicum } \\
\text { Linn. } \\
\end{array}$ & Babari & Herb & Flower & Paste, Juice & \begin{tabular}{|l}
$\begin{array}{l}\text { Scabbies, Ear } \\
\text { problem }\end{array}$ \\
\end{tabular} \\
\hline 19. & Dicotyledon & Lauraceae & $\begin{array}{l}\text { Lindera neesiana } \\
\text { Benth }\end{array}$ & Siltumur & Tree & Fruit & Raw, oil & $\begin{array}{l}\text { Gastric, low } \\
\text { appetite }\end{array}$ \\
\hline 20. & Dicotyledon & Leguminosae & Dolichos biflorus & Gahat & Herb & Seed & Cooked & Measles \\
\hline 21. & Dicotyledon & Leguminosae & \begin{tabular}{|l|} 
Trigonella \\
foenumgraceum
\end{tabular} & Methi & Herb & Seed & Powder & Cough \\
\hline 22. & Dicotyledon & Loranthaceae & $\begin{array}{l}\text { Viscum } \\
\text { articulatum }\end{array}$ & Hadchur & Shrub & Leaf & Paste & $\begin{array}{l}\text { Bone facture, } \\
\text { Back and } \\
\text { Body pain } \\
\end{array}$ \\
\hline 23. & Dicotyledon & Meliaceae & $\begin{array}{l}\text { Azadirachta indica } \\
\text { (A. Juss) }\end{array}$ & Neem & Tree & Leaf & Decoction & Fever \\
\hline 24. & Dicotyledon & Moraceae & Ficus religiosa L. & Peel & Tree & Root & Powder & Typhoid \\
\hline 25. & Dicotyledon & Myrtaceae & Psidium guajava & Ambak & Tree & Bark & Syrup & Diarrhoea \\
\hline 26. & Dicotyledon & Ranunculaceae & $\begin{array}{l}\text { Aconitum } \\
\text { palmatum }\end{array}$ & Bikhama & Herb & Root & Boiled & Fever \\
\hline 27. & Dicotyledon & Ranunculaceae & $\begin{array}{l}\text { Clematis } \\
\text { buchannania }\end{array}$ & $\begin{array}{l}\text { Pinashe } \\
\text { lahara }\end{array}$ & Climber & Root & Powder & Sinusities \\
\hline 28. & Dicotyledon & Rosaceae & Rubus ellipticus & Ainselu & Shrub & $\begin{array}{l}\begin{array}{l}\text { Young } \\
\text { shoot }\end{array} \\
\end{array}$ & Paste & $\begin{array}{l}\text { Tongue } \\
\text { Eczema. }\end{array}$ \\
\hline 29. & Dicotyledon & Rosaceae & Rosa brunonii & $\begin{array}{l}\begin{array}{l}\text { Bhansi } \\
\text { singe }\end{array} \\
\end{array}$ & Shrub & Root & Paste & Bone fracture. \\
\hline
\end{tabular}


Pradip Tamang and Nanda B. Singh

\begin{tabular}{|c|c|c|c|c|c|c|c|c|}
\hline 30. & Dicotyledon & Rosaceae & Pyrus pashia & Mel & Tree & \begin{tabular}{|l|} 
Fruit Bark, \\
Young \\
shoot
\end{tabular} & $\begin{array}{l}\text { Juice, } \\
\text { powder, Ra }\end{array}$ & Dysentery \\
\hline 31. & Dicotyledon & Rubiaceae & Rubia manjith & Majitho & Herb & $\begin{array}{l}\text { Whole } \\
\text { plant }\end{array}$ & Paste & Scabbies \\
\hline 32. & Dicotyledon & Rubiaceae & \begin{tabular}{|l|}
$\begin{array}{l}\text { Spermadictyon } \\
\text { suaveolens }\end{array}$ \\
\end{tabular} & Bhujchampa & Shrub & $\begin{array}{l}\text { Tuber, } \\
\text { root }\end{array}$ & Paste & Bone fracture. \\
\hline 33. & Dicotyledon & Rutaceae & $\begin{array}{l}\text { Citurs medica } \\
\text { Linn. }\end{array}$ & Bimra & Shrub & Root & Powder & $\begin{array}{l}\text { Intestinal } \\
\text { worm. }\end{array}$ \\
\hline 34. & Dicotyledon & Rutaeae & Citrus reticulate & Suntala & Tree & Root & Powder & Typhoid. \\
\hline 35. & Dicotyledon & Rutaceae & Citrus aurantifolia & Kagati & $\begin{array}{l}\text { Small } \\
\text { tree }\end{array}$ & Fruit & Juice & Vomiting \\
\hline 36 & Dicotyledon & Rutaceae & $\begin{array}{l}\text { Evodia frakinifolia } \\
\text { (Hook. F) }\end{array}$ & Khanakpa & $\begin{array}{l}\text { Small } \\
\text { tree }\end{array}$ & Fruit & Paste & Joint ache. \\
\hline 37. & Dicotyledon & Rutaceae & $\begin{array}{l}\text { Zanthoxylum } \\
\text { armatum }\end{array}$ & Timur & $\begin{array}{l}\text { Small } \\
\text { tree }\end{array}$ & $\begin{array}{l}\text { Fruit, } \\
\text { seed }\end{array}$ & Paste & Joint ache. \\
\hline 38. & Dicotyledon & Saxfragaceae & Bergenia ciliate & Pakhanbed & Herb & Leaf & Paste & Dog bite. \\
\hline 39. & Dicotyledon & Saxifragaceae & Astilbe rivularis & Thulo Okhat & Herb & Root & Powder & $\begin{array}{l}\text { back and body } \\
\text { pain }\end{array}$ \\
\hline 40. & Dicotyledon & Solanaceae & $\begin{array}{l}\text { Capsicum annum } \\
\text { Linn. }\end{array}$ & \begin{tabular}{|l} 
Dalle \\
Khuyrsani
\end{tabular} & Herb & Fruit & Raw & Gastric \\
\hline 41. & Dicotyledon & Solanaceae & Datura metel Linn. & Kalo Dhaturo & Herb & Seed & Raw & Dog bite \\
\hline 42. & Dicotyledon & Solanaceae & Datura sp. & \begin{tabular}{|l|} 
Seto \\
Dhaturo
\end{tabular} & Herb & Seed & Inhalation & Toothache. \\
\hline 43. & Dicotyledon & Solanaceae & Solanum indicum & Kande bean & herb & Seed & Raw & Tooth ache. \\
\hline 44. & Dicotyledon & Tenstroemiaceae & $\begin{array}{l}\text { Schinna wallichi } \\
\text { 'chois' }\end{array}$ & Chilaune & Tree & $\begin{array}{l}\text { Stem, } \\
\text { Root }\end{array}$ & & $\begin{array}{l}\text { Skin disease } \\
\text { 'khorna' }\end{array}$ \\
\hline 45. & Dicotyledon & Umbelliferae & Anethum sowa & Swoup & Herb & Seed & Decoction & $\begin{array}{l}\text { Internal } \\
\text { Eczema }\end{array}$ \\
\hline 46. & Dicotyledon & Umbelliferae & Carum copticum & Jwano & Herb & Seed & Powder & Gastric \\
\hline 47. & Dicotyledon & Urticaceae & Urtica dioca $\mathrm{L}$. & Sisnu & Herb & $\begin{array}{l}\begin{array}{l}\text { Leaf, } \\
\text { Young } \\
\text { shoot }\end{array} \\
\end{array}$ & Paste & Dog bite. \\
\hline 48. & Monocotyledon & Araceae & Acorus calamus & Bojho & Herb & Root & Paste & Scabbies \\
\hline 49. & Monocotyledon & bromeliaceae & Anana conqosus L. & Bhuikatahar & Herb & Leaf & Decoction & Fever. \\
\hline 50. & Monocotyledon & Gramineae & $\begin{array}{l}\text { Eleusinae } \\
\text { Coracana }\end{array}$ & Kodo & Shrub & Seed & Cooked & Measles \\
\hline 51. & Monocotyledon & Gramineae & \begin{tabular}{|l} 
Imperata \\
cylindrical
\end{tabular} & Siru & Shrub & Root & Powder & $\begin{array}{l}\text { Intestinal } \\
\text { worms. }\end{array}$ \\
\hline 52. & Monocotyledon & Gramineae & $\begin{array}{l}\text { Saccharum } \\
\text { officinarum }\end{array}$ & Ukhu & Grass & Stem & Juice & Jaundice \\
\hline 53. & Monocotyledon & G'ramineae & $\begin{array}{l}\text { Dendrocalamus } \\
\text { hamiltonii }\end{array}$ & Choya Bans & Tree & Buds & Paste & Pneumonia \\
\hline 54. & Monocotyledon & Liliaceae & Aloe barbadensis & Ghuikumari & Herb & Leaf & Juice & $\begin{array}{l}\text { Burnt, Stomach } \\
\text { disorder }\end{array}$ \\
\hline 55. & Monocotyledon & Musacae & Musa paradiasiaca & Kera & Tree & Stem & Juice & Snake bite. \\
\hline 56. & Monocotyledon & Zingiberaceae & $\begin{array}{l}\text { Amomum } \\
\text { Subulatum }\end{array}$ & Alainchi & Herb & Seeds & \begin{tabular}{|l} 
Powder, \\
Raw
\end{tabular} & $\begin{array}{l}\begin{array}{l}\text { Indigestion } \\
\text { vomiting }\end{array} \\
\end{array}$ \\
\hline 57. & Monocotyledon & Zingiberaceae & Concuma longa & Haledo & Herb & Rhizome & $\begin{array}{l}\text { Paste, Raw } \\
\text { Juice }\end{array}$ & Cough \\
\hline 58. & Monocotyledon & Zingiberaceae & Zingiber officinale & Aduwa & Herb & Rhizome & Paste & Vomiting \\
\hline 59. & Pteridophyta & Dryopteriadaceae & Tectaria coadunate & Kalo niguro & Fern & Root & Powder & Dysentery \\
\hline 60. & Pteridophyta & Nephrolepidaceae & $\begin{array}{l}\text { Nephrolepis } \\
\text { auriculata }\end{array}$ & Paniamala & $\begin{array}{l}\text { Tree } \\
\text { fern }\end{array}$ & Tuber & Raw & Fever, Tonsil. \\
\hline 61. & Pteridophyta & Polypodaceae & Dryopteris filixmas & Hade unyu & Tree fern & Leaf & Juice & Cut wound \\
\hline
\end{tabular}




\section{Indigenous knowledge system}

Indigenous knowledge of the Lapcha of Fikkal VDC was very rich and it fully supported the livelihood of people. They used their indigenous knowledge in their daily activities like food processing, agriculture, biodiversity conservation, preparation and use of domestic materials, storage of food grains and use of manure and organic insecticides and pesticides. The use of their indigenous knowledge is outlined as below.

- Indigenous knowledge system in preparation and selection of wild edible food like simal tarul (Manihot utilissima), Giththa (Dioscorea sp.), Sissnu (Urtica dioca) and Mushroom.

- They also have sound knowledge in preparing dyes of different colours.

- The Lapcha people are very closely related with bamboo so they are highly skilled in using it for preparing different things needed in daily life like building houses, bridges, handicrafts, bows, arrows, furniture, baskets, fences, firewood, flutes etc.

- They have good knowledge for preparation and use of insecticides in agriculture and prevent livestock from pests. The dust powder of Neem (Bhumea lacera) helps to preserve the seeds of wheat, maize and beans. The fresh and matured tobacco (Nicotiana tabaca) leaves is steamed and firstly grinded in the 'Okhali' (a wooden grinder) in order to extract juice make juice and made into a solution with water at a ratio of 1:2 (tobacco: water). This solution is sprayed over infected plants.

- They also have sound knowledge on conserving bio-diversity as they preserve their own forest where medicinal plants, wild edible food and animals are preserved.

\section{DISCUSSION}

The local Lapcha people of Fikkal VDC have been using both wild and domesticated plants and animals in their traditional medicinal practices since time immemorial. They have a rich tradition, culture and indigenous knowledge to support the utilization of various plants and animal species for medicinal purposes. The present study unveiled that they used about 19 animal species both wild and domesticated belonging to 10 order and 13 families and 61 species of medicinal plants belonging to 39 families, 58 genera for medicinal purposes for their traditional healing practices where plant species were dominant over animal species.

Analysis of data showed that 19 animal species recorded from the study area was used in the treatment of 21 different diseases/ailments by the local Lapchas using their indigenous knowledge. Among the 19 animal species, 11 species were mammals, 1 species each of Aves, Reptiles, Amphibian and Pisces and 4 species were invertebrates. For different medicinal purpose different parts as well as products of animal species such as honey, teeth, bile, milk, meat, born, hide, brain, hoof, foetus, saliva, stomach, e.g., fat etc as well as the whole organism were used. The most frequently used animal part was bile for 4 medical remedies followed by bone for 3 remedies and meat, egg, fat, milk, honey for 2 remedies each. Among the total animal species used for medicinal purposes, 6 species of them were used to cure disease of respiratory track and for cuts and wounds, 5 species each for gastro-intestinal and skeletal problems and 1 each for dog bite, mushroom's poison, ring worm, snake bite and pungent smell from body (Thangne); respectively. Most of the species were used raw for medicine and some of the animal species were used to treat more than one disease. Medicines were basically administered in two ways, orally for internal medication (15 remedies) and applying for external medication (11 remedies).

The cooked meat and alcohol of meat of Canis aureus was also reported by Kaundinya (1998), Acharya (1999), Dhakal (2004) and Thapa (2008) in their study for the treatment of rheumatism. However, Negi and Palyal (2007) reported the use of meat of this species in curing paralysis and arthritis and blood for asthma. Thakur (2008) reported the use of urine of this species in treatment of insanity and epilepsy. The stomach of Hystrix brachyura used for curing Asthma was also reported by Kaundinya (1998), Acharya (1999), Tamang (2003), Dhakal (2004), Koirala (2004), Negi and Palyal (2007) and Thapa (2008). Kafle (2000), Koirala (2004) and Thapa (2008) reported the use of Gallus gallus domesticus for curing burn and Kaundinya (1998) and Dhakal (2004) reported its use for bone fracture. The honey of Apis cerana was reported for curing cough, body and back pain was supported by findings of Koirala (2004), Tamang (2003) and Thapa (2008). Thapa (2008) reported the use of Anadenus sp. in treatment of fractured bone.

The documented 61 species of medicinal plants have been used for the treatment of 36 different ailment/diseases. Among these 61 medicinal plants species, $77.04 \%$ belongs to dicotyledons, $18: 03 \%$ monocotyledons and $4.91 \%$ pteridophytes. The primary source of medicinal plants in terms of number of species were herbs (50.82\%), followed by trees $(26.23 \%)$, shrubs $(8.19 \%)$, climber $(6.56 \%)$, fern $(4.91 \%)$ and grass $(3.27 \%)$ of total species. The different plant parts used for medicinal preparations were roots, leaves, seeds, bark, rhizome, flower, young shoots, stem, tuber, buds and sometimes whole plant is also used. The most repeatedly used plant part was the root (14 medical 
remedies) followed by leaf ( 13 medical remedies), seed (11 medical remedies) and fruits (8 medical remedies). The frequently treated disease or ailments in their traditional healing system are respiratory tract infections, gastro-intestinal ailments, skeleto-muscular problems and dermatological infections.

The medicinal plant species documented in the study area to have folk medicines utility are also supported by the findings of other researcher. Ghimire (1999), Rai (2003), Tamang (2003) also reported the use of Aloe barbadensis for treating the burnt wound but Ramana (2008) reported the use of this species for curing eye infection. Thapa (2008) reported the use of Swertia chirata in controlling fever. Ghimire (1999) reported use of Swertia chirata as antihelminthic drug and Rai (2003) reported its use in treating Hypertension and Diabetes. Oli (2003) reported the use of the same species for fever, pneumonia, cough and diarrhoea/dysentery. Also Pandey (2006) reported the use of Swertia species for fever, jaundice, indigestion, cough, cold, blood and gall bladder problems. The study of Oli (2003), Thapa (2008) reported the use of Artemisia indica in cuts and wounds to stop bleeding and Joshi and Joshi (2007) reported its use in curing itching. However, Ghimire (1999), Rai (2003) reported its use in helminthic cure and nervous fresher respectively and Tamang (2003) for nausea problem and intestinal worms. Pandey (2006) reported the use of same species for cough and cold. Tamang (2003) Oli (2003) reported the use of Imperata cylindrica to cure intestinal worms. However, Rai (2003) has reported the use of this species for curing piles, diarrhea. Thapa (2008) reported the use of Lepidium sativum in curing back and body pains. However, Rai (2003) has reported the use of this species in curing asthma, cough, poles and diuretic. The use of flower of Rhododendron arboreum for fish bone prick was also reported by Subedi (1998), Karki (2001), Gurung (2002), Shrestha and Dhillion (2003), Oli (2003), and Thapa (2008). Also the use of the same plant has been reported by Rai (2003) and Thapa (2008) for curing dysentery. The use of Viscum articulatum in curing bone fracture was also reported by Ghimire (1999), Oli (2003) and Rai (2003).

\section{CONCLUSION}

The Lapcha community of the study area had a sound knowledge on the usage of locally available animals and plant species for traditional healing system as medicines. The plant species were used mostly in comparison to animal species. The local healers (Yaba/ Yama, Boongthing/Mun) were the most popular one in the village for utilizing the medicinal plants and animals in the traditional healing practices. Along with them the Lamas, elderly people, women and others also had knowledge on traditional medicine gained from their experiences and practices done from generations and used them as home remedy.

With the rise of younger generations and increment of the modern health facilities, the traditional healing practices have been shadowed a bit. The inclination of younger generation towards modern medicines is also due to lack of awareness and reluctance of older generation in sharing their knowledge with them which poses a great threat to its existence. Despite such gloomy facts, still many of villagers depended on traditional medicine for their primary health care and cure simple diseases/ ailments such as fever, diarrhoea, dysentery, cold, cough, cut and burnt wound etc at home. Therefore, the following recommendations are made in the hope of popularizing the traditional medicinal systems and uplifting it with proper management and scientific approach helping the Lapcha community to upgrade their health standard in low cost effectively and efficiently.

- Create awareness about the importance of medicinal plants and animals with the feeling of ownership.

- Provide expertise help and training for local people to promote preservation and conservation of medicinal plants and animals for sustainable use.

- Increase frequency of research and investigation of indigenous knowledge system on biodiversity conservation.

- Motivate the traditional healers for dissemination of their knowledge to youngsters for benefit of their community and mankind at large.

- The bio chemical analysis of the plants and animal parts should be done for its efficacy and verification and their patent rights should be taken to stop their abuse.

- Lastly, the government should recognize the indigenous knowledge system present in the indigenous communities and encourage preserving it by bringing comprehensive plans and policies in national level.

\section{ACKNOWLEDGEMENTS}

We are highly indebted to Central Department of Zoology, T.U. Kirtipur for the cooperation extended during our research period. We are grateful to Rong Sejum Thi (Lapcha Uththan Manch), all the local people of Fikkal VDC who shared their rich indigenous knowledge on the usage of medicinal plants and animals. We are also indebted to Mr. Bir Bahadur Lapcha and Prem Bahadur Lapcha for their help and cooperation during the field visit. 


\section{REFERENCES}

Bhattarai, N.K. 1992. Medical ethnobotany in the Karnali zone, Nepal. Economic Botany 36(3): 257-261.

CBS. 2002. Population of Nepal, Population census 2001- Selected Tables on Castel Ethnicity, Mother tongue and Religion. Central Bureau of Statistics, Kathmandu, Nepal.

CBS. 2003. Statistical Year Book of Nepal 2003. Central Bureau of Statistics, Kathmandu, Nepal.

CBS. 2011. Population of Nepal, Population census 2011. Central Bureau of Statistics, Kathmandu, Nepal.

Chaudhary, R.P. 1998. Biodiversity in Nepal: Status and Conservation S.Devi, Saharanpur, India.

Dhakal, N. 2004. Ethnobiology of the Magars A Case study of Trimure VDC of Palpa. M.Sc. Thesis, Central Department of Zoology T.U., Kirtipur, Kathmandu, Nepal.

Ghimire, K. 1999. Ethnomedicobotany of Tharu tribe of Nawalparasi District. Study report submitted to DNPWC, Kathmandu, Nepal.

Gurung, A. 2002. A study on medicinal Plants and their Traditional Uses in Chitre VDC (Parbat) and Bhadauri/Tamagi VDC (Kaski), Western Nepal. M.Sc. Thesis, Central Department of Botany, T.U., Kirtipur, Kathmandu, Nepal.

HMGN. 1970. Medicinal Plants of Nepal, Bulletin of the Department of Medicinal Plants, No.3, HMG of Nepal, Ministry of Forest and Soil Conservation, Kathmandu, Nepal.

HMGN. 1984. Medicinal Plants of Nepal (Supplement volume), Bulletin of Department of Medicinal plants No. 10, HMG, Ministry of Forest and Soil Conservation, Kathmandu, Nepal.

Joshi, A.R. and Joshi, K. 2007. Ethnomedicinal Plants Used against skin Diseases in Some Villages of Kali Gandaki, Bagmati and Todi Likhu Wqatersheds of Nepal. Ethnobotanical leaflets 11:235-246.

Kafle, G. 2000. Ethnobiology of the Tharus: A case study of Gardi VDC of Chitwan. M.Sc. Thesis, Central Department of Zoology, T.U., Kirtipur, Kathmandu, Nepal.

Karki, R. 2001. Medicinal Plant and Traditional Medicinal Practices in Ugrachandi and Tukucha VDC of Kavrepalanchowk District. M.Sc. Thesis, Central Department of Botany, T.U., Kirtipur, Kathmandu, Nepal.

Kaundinya, D.P. 1998. Ethnobiology of the Kumals. A case study of Chirtungdhara VDC of Palpa district. M.Sc. Thesis, Central Department of Zoology, T.U., Kirtipur, Kathmandu, Nepal.
Koirala, A. 2004. Ethnobiology of the Musahars: A case study of Bachhauli VDC of Chitwan District. M.Sc. Thesis, Central Department of Zoology, T.U., Kirtipur, Kathmandu, Nepal.

Malla, S.B. and Shakya, P.R. 1984/85. Medicinal Plants. Nepal Natures Paradise. pp.261-297.Craftmans Press, Bangkok.

Negi, C.S. and Palyal, V.S. 2007. Traditional in medicine and Rituals by the Shoka Tribes of Districk Pithoragraph, Uttaranchal, India. Ethno-Med. 1(1): 47-54).

Oli, B.R. 2003. Ethno-Medicinal Uses of Plants among the Limbus of Hellock Area of Tapethok VDC, Taplejung, Nepal. Botanica Orientalis. pp 112115.

Pandey, M.R. 2006. Use of medicinal Plants in Traditional Tibetan Therapy SysTem in Upper Mustang, Nepal. Out Nature 4:69-82.

Rai, M.B. 2003. Medicinal Plants of Tehrathum District, Eastern Nepal. Our Nature 1:42-48.

Shrestha, P.M. and Dhillion S.S. 2003. Medicinal Plant diversity and use in the highlands of Dolakha, District, Nepal. Journal of Ethnopharmacology, 86: 81-96.

Subedi, B.P. 1998. Participatory Utilization and Conservation of Medicinal and Aromatic Plants. A case from Western Nepal Himalayan. Medicinal Plants: A global Heritage proceedings of the International Conference on Medicinal Plants for survival.

Tamang, G. 2003. An Ethnobiological study of the Tamang People. Our nature 1:37.41.

Tamsang, K.P. 1983. The Unknown and Untold Reality about the Lapchas. Published by Luyangsang Tamsang Luen Sun offset printing Co. Ltd. Hong Kong.

Thakur, A.N. 2004. Therapeutic use of urine in early Indian medicine. Indian Journal of History of Science, 29(4) pp: 415-427.

Thapa, S. 2008. Medico-ethnobiological Knoweldge of the Magars: A case study of Salija VDC, Parbat District. M.Sc. Thesis, Central Department of Environmental Science, T.U., Kirtipur, Kathmandu, Nepal.

Tiwari, N.N. 1994. Wild Relatives of Cultivated Medicinal and Aromatic Plants in Nepal, Proceedings of National Conference on Wild Relatives of Cultivated Plants in Nepal. pp: 141-148. 